\title{
Application of Boosting Regression Trees to Preliminary Cost Estimation in Building Construction Projects
}

\author{
Yoonseok Shin \\ Department of Plant and Architectural Engineering, Kyonggi University, Gwanggyosan-ro 154-42, Yeongtong-gu, \\ Suwon, Gyeonggi-do 443-760, Republic of Korea \\ Correspondence should be addressed to Yoonseok Shin; shinys@kgu.ac.kr
}

Received 8 October 2014; Revised 11 December 2014; Accepted 7 January 2015

Academic Editor: Rahib H. Abiyev

Copyright (C) 2015 Yoonseok Shin. This is an open access article distributed under the Creative Commons Attribution License, which permits unrestricted use, distribution, and reproduction in any medium, provided the original work is properly cited.

\begin{abstract}
Among the recent data mining techniques available, the boosting approach has attracted a great deal of attention because of its effective learning algorithm and strong boundaries in terms of its generalization performance. However, the boosting approach has yet to be used in regression problems within the construction domain, including cost estimations, but has been actively utilized in other domains. Therefore, a boosting regression tree (BRT) is applied to cost estimations at the early stage of a construction project to examine the applicability of the boosting approach to a regression problem within the construction domain. To evaluate the performance of the BRT model, its performance was compared with that of a neural network (NN) model, which has been proven to have a high performance in cost estimation domains. The BRT model has shown results similar to those of NN model using 234 actual cost datasets of a building construction project. In addition, the BRT model can provide additional information such as the importance plot and structure model, which can support estimators in comprehending the decision making process. Consequently, the boosting approach has potential applicability in preliminary cost estimations in a building construction project.
\end{abstract}

\section{Introduction}

In building construction, budgeting, planning, and monitoring for compliance with the client's available budget, time, and work outstanding are important [1]. The accuracy of the construction cost estimation during the planning stage of a project is a crucial factor in helping the client and contractor with the adequate decision making and for the successful completion of the project [2-5]. However, there is a problem in that it is difficult to quickly and accurately estimate the construction costs at the early stage because the drawings and documentation are generally incomplete [6]. Machine learning approaches can be applied to alleviate this problem. Machine learning has some advantages over the human-crafted rules for data driven works, that is, accurate, automated, fast, customizable, and scalable [7].

Cost estimating approaches using a machine learning technique such as a neural network (NN) or support vector machine (SVM) have received significant attention since the early 1990s for accurately predicting the construction costs under a limited amount of project information. The NN model [1, 8-11] and the SVM model [12-16] were developed for predicting and/or estimating the construction costs. Although applying an NN to construction cost estimations has been very popular and has shown superior accuracy over other competing techniques [2, 4, 17-21], it has several disadvantages, such as a lack of self-learning and a timeconsuming rule acquisition process [14]. A SVM, introduced by Vapnik [22], has attracted a great deal of attention because of its capacity for self-learning and high performance in generalization; moreover, it has shown the potential for utilization in construction cost estimations $[5,13,14,16,23$, 24]. However, the SVM approach requires a great deal of trial and error to determine a suitable kernel function [14]. Moreover, SVM models have a high level of algorithmic complexity and require extensive amounts of memory [25].

Among the recent machine learning techniques, the boosting approach, which was developed by Freund and Schapire [26], who also introduced the AdaBoost algorithm, has become an important application in machine learning and predicting models [27]. The boosting approach provides an effective learning algorithm and strong boundaries in 
terms of the generalization performance [28-31]. Compared with competing techniques used for prediction problems, the performance of the boosting approach is superior to that of both a NN [32] and a SVM [33]. It is also simple, easy to program, and has few parameters to be tuned $[31,34,35]$. Because of these advantages, the boosting approach has been actively utilized in various domains. In the construction domain, some studies have attempted to apply this approach to the classification problem (for predicting a categorical dependent variable), such as the prediction of litigation results [27] and the selection of construction methods [31, 36]. However, there have been no efforts to do so for regression problems (for predicting a continuous dependent variable), such as construction cost estimation.

In this study, the boosting regression tree (BRT) is applied to the cost estimation at the early stage of a construction project to examine the applicability of the boosting approach for a regression problem within the construction domain. The BRT in this study is based on the module of a stochastic gradient boosting tree, which was proposed by Friedman (2002) [37]. It was developed as a novel advance in data mining that extends and improves the regression tree using a stochastic gradient boosting approach. Therefore, it has advantages of not only a boosting approach but also a regression tree, that is, high interpretability, conceptual simplicity, computational efficiency, and so on. The boosting approach can especially adopt the other data mining techniques, that is, a NN and SVM, as well as decision tree, as base learner. This feature matches up to the latest trends in the field of fusion of computational intelligence techniques to develop efficient computational models for solving practical problems.

In the next section, the construction cost estimation and its relevant studies are briefly reviewed. In the third section, the theory of a BRT and a cost estimation model using a BRT are both described. In the fourth section, the cost estimation model using a BRT is applied to a dataset from an actual project of a school building construction in Korea and is compared with that of an NN and an SVM. Finally, some concluding remarks and suggestions for further study are presented.

\section{Review of Cost Estimation Literature}

Raftery [38] categorized the preliminary cost estimation system used in building construction projects into three generations. The first generation of the system was a method from the late 1950s to the late 1960s that utilized the unitprice. The second generation of the system, which was developed from the middle of the 1970s, was a statistical method using a regression analysis according to propagating personal computers. The third generation of the system is a knowledge-based artificial intelligence method from the early 1980s. However, based on the third generation, Kim [39] also separated a fourth generation based on machine learning techniques such as a NN and SVM. The author showed an outstanding performance in construction cost estimation, although much remains to be resolved, for example, the complexity of the parameter settings.

We believe that the boosting approach can be a nextgeneration cost estimation system at the early stage of a

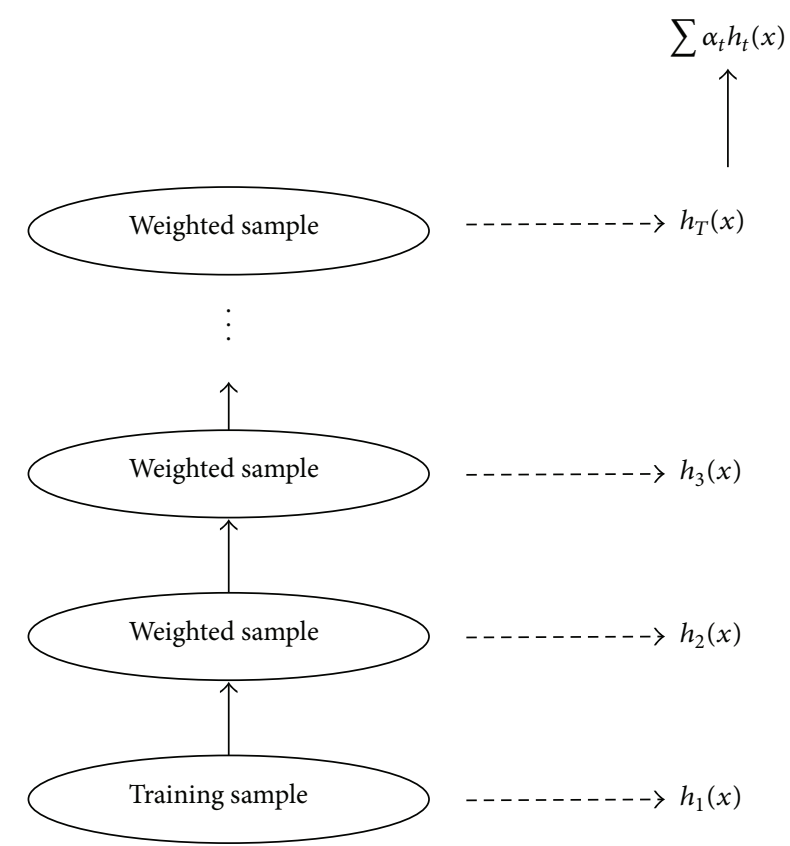

FIGURE 1: Schematic of a boosting procedure.

construction project. In the prediction problem domain, combining the predictors of several models often results in a model with improved performance. The boosting approach is one such method that has shown great promise. Empirical studies have shown that combining models using the boosting approach produces a more accurate regression model [40]. In addition, the boosting approach can be extensively applied to prediction problems using an aforementioned machine learning technique such as a NN and SVM, as well as decision trees [27]. However, the boosting approach has never been used in regression problems of the construction domain, including cost estimations, but has been actively utilized in other domains, such as remote aboveground biomass retrieval [41], air pollution prediction [42], software effort estimation [43], soil bulk density prediction [44], and Sirex noctilio prediction [45]. In this study, we examine the applicability of a BRT for estimating the costs in the construction domain.

\section{Boosting Regression Trees}

Because of the abundance of exploratory tools, each having its own pros and cons, a difficult problem arises in selecting the best tool. Therefore, it would be beneficial to try to combine their strengths to create an even more powerful tool. To a certain extent, this idea has been implemented in a new family of regression algorithms referred to under the general term of "boosting." Boosting is an ensemble learning method for improving the predictive performance of a regression procedure, such as the use of a decision tree [46]. As shown in Figure 1, the method attempts to boost the accuracy of any given learning algorithm by fitting a series of models, each having a low error rate, and then combining them into 


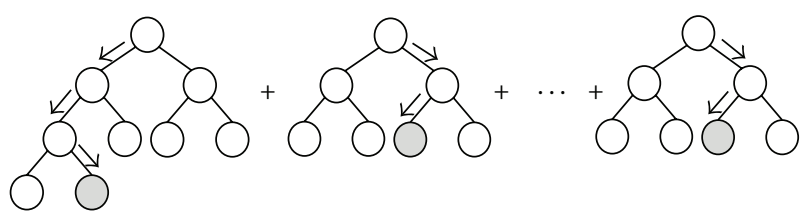

FIgURE 2: Gradient boosted decision tree ensemble.

an ensemble that may achieve better performance [36, 47]. This simple strategy can result in a dramatic improvement in performance and can be understood in terms of other wellknown statistical approaches, such as additive models and a maximum likelihood [48].

Stochastic gradient boosting is a novel advance to the boosting approach proposed by Friedman [37] at Stanford University. Of the previous studies [26, 49-51] related to boosting for regression problems, only Breiman [50] alludes to involving the optimization of a regression loss function as part of the boosting algorithm. Friedman [52] proposed using the connection between boosting and optimization, that is, the gradient boost algorithm. Friedman [37] then showed that a simple subsampling trick can greatly improve the predictive performance of stochastic gradient boost algorithms while simultaneously reducing their computational time.

The stochastic gradient boost algorithm proposed by Friedman [37] uses regression trees as the basis functions. Thus, this boosting regression tree (BRT) involves generating a sequence of trees, each grown on the residuals of the previous tree [46]. Prediction is accomplished by weighting the ensemble outputs of all regression trees, as shown in Figure 2 [53]. Therefore, this BRT model inherits almost all of the advantages of tree-based models, while overcoming their primary disadvantages, that is, inaccuracies [54].

In these algorithms, the BRT approximates the function $F(x)$ as an additive expansion of the base learner (i.e., a small tree) [43]:

$$
F(x)=F_{0}(x)+\beta_{1} F_{1}(x)+\beta_{2} F_{2}(x)+\cdots+\beta_{m} F_{m}(x) .
$$

A single base learner does not make sufficient prediction using the training data, even when the best training data are used. It can boost the prediction performance using a series of base learners with the lowest residuals.

Technically, BRT employs an iterative algorithm, where, at each iteration $m$, a new regression tree $h\left(x ;\left\{R_{l m}\right\}_{l}^{L}\right)$ partitions the $x$-space into $L$-disjoint regions $\left\{R_{l m}\right\}_{l}^{L}$ and predicts a separate constant value in each one [54]:

$$
h\left(x ;\left\{R_{l m}\right\}_{l}^{L}\right)=\sum_{l-1}^{L} \bar{y}_{l m} \mid\left(x \in R_{l m}\right) .
$$

Here $\bar{y}_{l m}=\operatorname{mean}_{x_{i} \in R_{l m}}\left(\tilde{y}_{i m}\right)$ is the mean of pseudo-residuals (3) in each region $R_{l m}$ induced at the $m$ th iteration $[37,54]$ :

$$
\tilde{y}_{i m}=-\left[\frac{\partial \Psi\left(y_{i}, F\left(x_{i}\right)\right)}{\partial F\left(x_{i}\right)}\right]_{F(x)=F_{m-1}(x)} .
$$

The current approximation $F_{m-1}(x)$ is then separately updated in each corresponding region $[37,54]$ :

$$
F_{m}(x)=F_{m-1}(x)+v \cdot \gamma_{l m} \mid\left(x \in R_{l m}\right),
$$

where

$$
\gamma_{l m}=\underset{\gamma}{\arg \min } \sum_{x_{i} \in R_{I m}} \Psi\left(y_{i}, F_{m-1}\left(x_{i}\right)+\gamma\right) .
$$

The "shrinkage" parameter $v$ controls the learning rate of the procedure.

This leads to the following BRT algorithm for generalized boosting of regression trees [37].

(1) Initialize $F(x), F_{0}(x)=\arg \min _{\Upsilon} \sum_{i-1}^{N} \Psi\left(y_{i}, \gamma\right)$.

(2) For $m=1$ to $M$ do

(3) Select a subset randomly from the full training dataset,

$$
\{\pi(i)\}_{l}^{N}=\text { rand_perm }\{i\}_{l}^{N} .
$$

(4) Fit the base learner,

$$
\tilde{y}_{\pi(i) m}=-\left[\frac{\partial \Psi\left(y_{\pi(i) m}, F\left(x_{(i)}\right)\right)}{\partial F(x(i))}\right]_{F(x)=F_{m-1}(x)}, \quad i=1, \widetilde{N} .
$$

(5) Compute the model update for the current iteration,

$$
\left\{R_{l m}\right\}_{I}^{L}=L-\text { terminal node tree }\left(\left\{\widetilde{y}_{\pi(i) m}, x_{\pi(i)}\right\}_{l}^{\widetilde{N}}\right) .
$$

(6) Choose a gradient descent step size as,

$$
\gamma_{l m}=\underset{\gamma}{\arg \min } \sum_{x_{(i)} \in R_{l m}} \Psi\left(y_{\pi(i)}, F_{m-1}\left(x_{\pi(i)}\right)+\gamma\right) .
$$

(7) Update the estimate of $F(x)$ as,

$$
F_{m}(x)=F_{m-1}(x)+v \cdot \gamma_{l m} \mid\left(x \in R_{l m}\right) .
$$

(8) end For.

There are specific algorithms for several loss criteria including least squares: $\psi(y, F)=(y-F)^{2}$, least-absolute deviation: $\psi(y, F)=|y-F|$, and Huber- $M: \psi(y, F)=(y-$ $F)^{2}|(|y-F| \preccurlyeq \delta)+2 \delta(|y-F|-\delta / 2)|(|y-F|>\delta)$ [37]. The BRT applied in this study adopts the least squares for loss criteria as shown in Figure 3.

\section{Application}

4.1. Determining Factors Affecting Construction Cost Estimation. In general, the estimation accuracy in a building project is correlated with the amount of project information available regarding the building size, location, number of stories, and so forth [55]. In this study, the factors used for estimating the construction costs are determined in two steps. First, a 
TABLE 1: Factors in construction cost estimation.

\begin{tabular}{|c|c|c|c|c|}
\hline Description & Min. & Max & Average & Remark \\
\hline \multicolumn{5}{|l|}{ Input } \\
\hline \multirow[t]{2}{*}{ Budget } & & (1) BTL & & Nominal \\
\hline & & $\begin{array}{l}\text { (2) National finance } \\
\text { (1) Elementary }\end{array}$ & & \multirow{3}{*}{ Nominal } \\
\hline \multirow[t]{3}{*}{ School levels } & & (2) Middle & & \\
\hline & & (3) High & & \\
\hline & & (1) Existing & & \multirow{3}{*}{ Nominal } \\
\hline \multirow[t]{2}{*}{ Land acquisition } & & (2) Building lots & & \\
\hline & & (3) Green belts & & \\
\hline Class number & 12 & 48 & 31 & Numerical \\
\hline Building area $\left(\mathrm{m}^{2}\right)$ & 1,204 & 3,863 & 2,694 & Numerical \\
\hline Gross floor area $\left(\mathrm{m}^{2}\right)$ & 4,925 & 12,710 & 9,656 & Numerical \\
\hline Storey & 3 & 7 & 4.7 & Numerical \\
\hline Basement floor (storey) & 0 & 2 & 0.5 & Numerical \\
\hline Floor Height $(\mathrm{m})$ & 3.3 & 3.6 & 3.5 & Numerical \\
\hline \multicolumn{5}{|l|}{ Output } \\
\hline $\begin{array}{l}\text { Total construction cost } \\
\text { (thousand KRW) }\end{array}$ & $4,334,369$ & $14,344,867$ & $8,288,008$ & Numerical \\
\hline
\end{tabular}

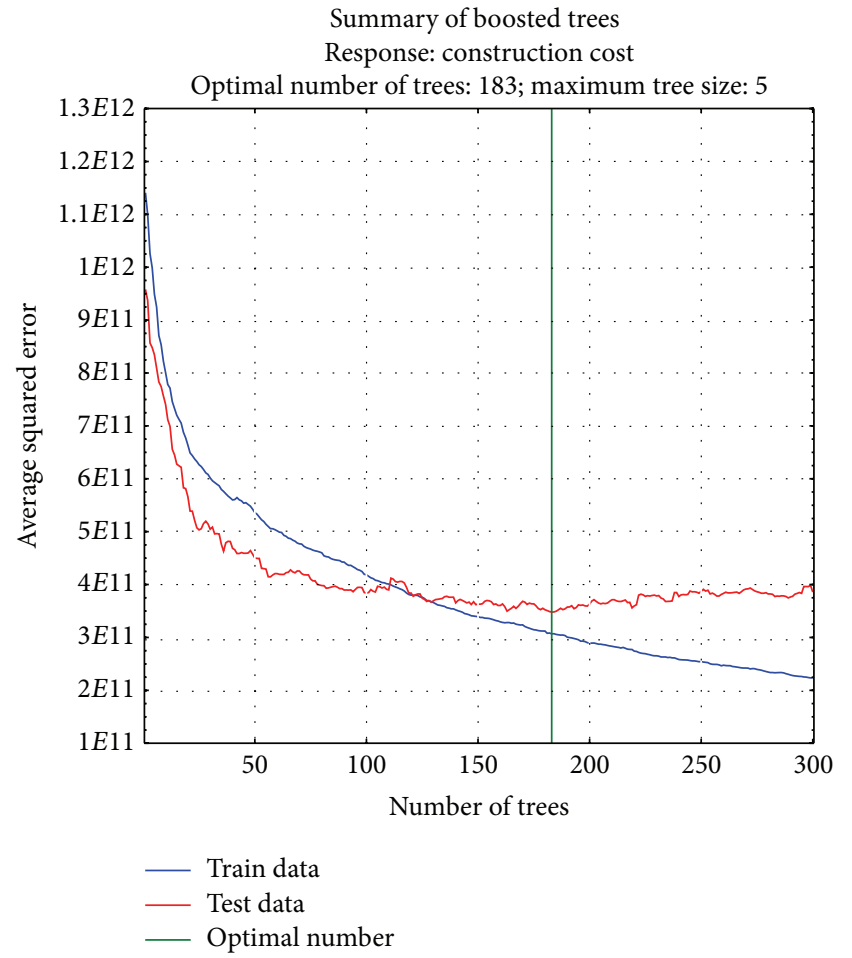

FIgURE 3: Training results of BRT.

list of factors affecting the preliminary cost estimation was made by reviewing previous studies $[2,3,8,12,14,20,23$, $55,56]$. Lastly, appropriate factors were selected from this list by interviewing practitioners who are highly experienced in construction cost estimation in Korea. Consequently, nine factors (i.e., input variables) were selected for this study, as shown in Table 1.

4.2. Data Collection. Data were collected from 234 completed school building projects executed by general contractors from 2004 to 2007 in Gyeonggi Province, Korea. These cost data were only the direct costs of different school buildings, such as elementary, middle, and high schools, without a markup as shown in Figure 4. According to the construction year, the total construction costs were converted using the Korean building cost index (BCI); that is, the collected cost data were multiplied by the BCI of the base year of 2005 (BCI = 1.00). The collected cost data of 217 school buildings were randomly divided into 30 test datasets and 204 training datasets.

4.3. Applying BRT to Construction Cost Estimation. In this study, the construction cost estimation model using a BRT was tested through application to real building construction projects. The construction costs were estimated using the BRT as follows. (1) The regression function $\widehat{F}(x)$ was trained using training data. In the dataset, the budget, school levels, gross floor area, and so on were allocated to each $x_{i}$ of the training set. Each result, that is, the actual cost, was allocated to $y_{i}$. (2) After the training was completed according to the parameters such as the learning (shrinkage) rate, the number of additive trees, and the maximum and minimum number of levels, the series of trees $\widehat{F}(x)$ which maps $x$ to $y$ of training data set $\left(y_{i}, x_{i}\right)$ with minimized loss function $\Psi\left(y_{i}, F\left(x_{i}\right)\right)$ was found. (3) The expected value of $\widehat{F}(x)$, that is, the expected cost, was calculated for a new test dataset $\left(y_{j}, x_{j}\right)$.

The construction cost estimation model proposed in this study was constructed using "STATISTICA Release 7." STATISTICA employs an implementation method usually 


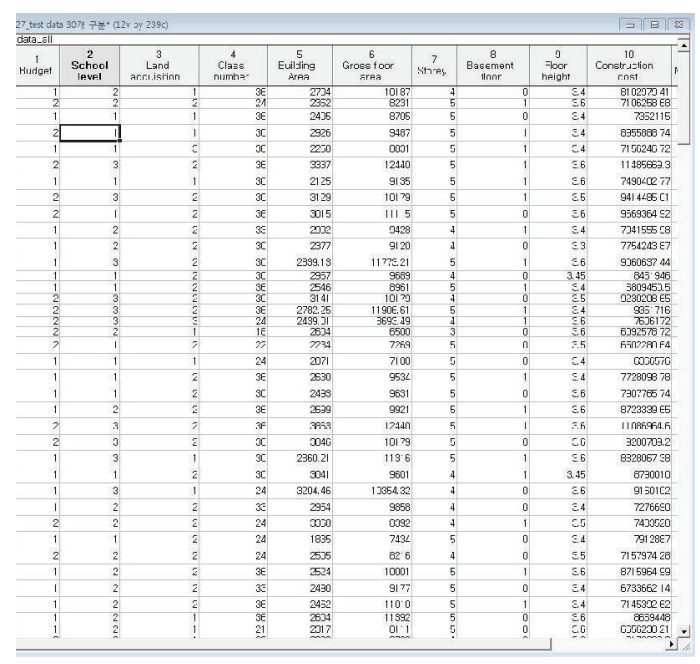

FIgURE 4: Fragment of cost dataset.

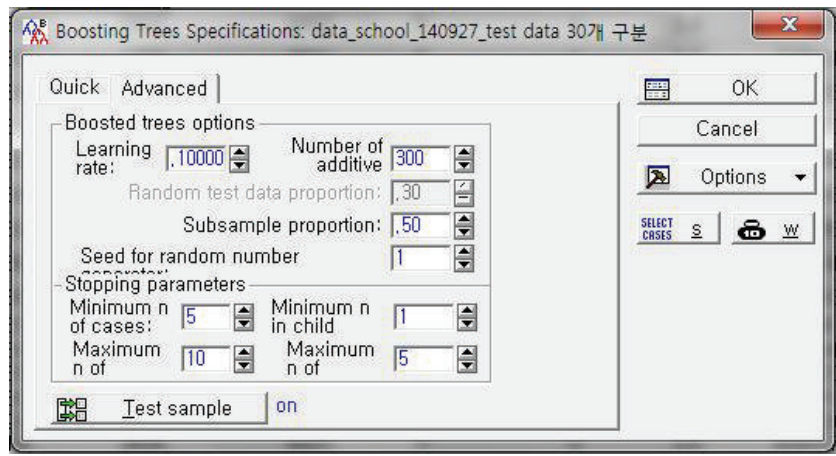

FIGURE 5: Parameter setting for BRT.

referred to as a stochastic gradient boosting tree by Friedman $(2002,2001)$ [37, 52], also known as TreeNet (Salford Systems, Inc.) or MART (Jerill, Inc.). In this software, a stochastic gradient booting tree is used for regression problems to predict a continuous dependent variable [57]. To operate a boosting procedure in STATISTICA, the parameter settings, that is, the learning rate, the number of additive trees, the proportion of subsampling, and so forth, are required. Firstly, the learning rate was set as 0.1. It was found that small values, that is, values under 0.1 , lead to much better results in terms of the prediction error [52]. We empirically obtained the other parameters, which are shown in Figure 5. As a result, the training result of the BRT showed that the optimal number of additive trees is 183 and the maximum size of tree is 5 , as shown in Figure 3.

4.4. Performance Evaluation. In general, the cost estimation performance can be measured based on the relationship between the estimated and actual costs [56]. In this study, the performance was measured using the Mean Absolute Error Rates (MAERs), which were calculated using

$$
\text { MAERs }=\frac{\left(\sum\left|\left(\left(C_{e}-C_{a}\right) / C_{a}\right) \times 100\right|\right)}{n},
$$

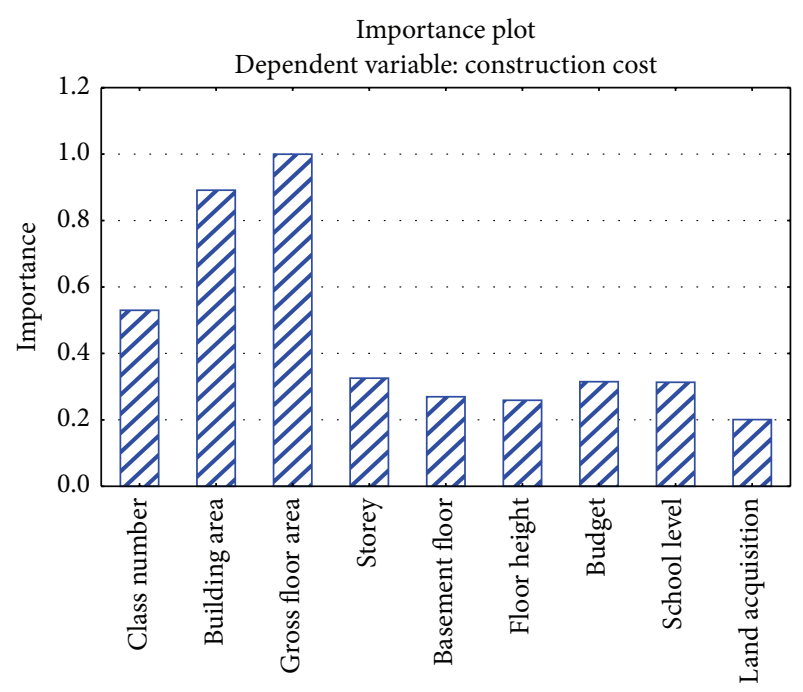

FIGURE 6: Importance plot of dependent variables.

where $C_{e}$ is the estimated construction costs by model application, $C_{a}$ is the actual construction costs collected, and $n$ is the number of test datasets.

To verify the performance of the BRT model, the same cases were applied to a model based on a NN and the results compared. We chose the NN model because it showed a superior performance in terms of cost estimation accuracy in previous studies $[2,5,14]$. "STATISTICA Release 7 " was also used to construct the NN model in this study. To construct a model using a $\mathrm{NN}$, the optimal parameters have to be selected beforehand, that is, the number of hidden neurons, the momentum, and the learning rate for the NN. Herein, we determined the values from repeated experiments.

\section{Results and Discussion}

5.1. Results of Evaluation. The results from the 30 test datasets using a BRT and a NN are summarized in Tables 2 and 3 . The results from the BRT model had MAERs of 5.80 with $20 \%$ of the estimates within $2.5 \%$ of the actual error rate, while $80 \%$ were within $10 \%$. The NN model had MAERs of 6.05 with $10 \%$ of the estimates within $2.5 \%$ of the actual error rate, while $93.3 \%$ were within $10 \%$. In addition, the standard deviations of the NN and BRT models are 3.192 and 3.980, respectively, as shown in Table 4.

The MAERs of two results were then compared using a $t$-test analysis. The MAERs of the two results are statistically similar, although there are differences between them. As the null hypothesis, the MAERs of the two results are all equal $\left(H_{0}: u_{D}=0\right)$. The $t$-value is 0.263 and the $P$ value is 0.793 $(>0.05)$. Thus, the null hypothesis is accepted. This analysis shows that the MAERs of the two results are statistically similar.

The BRT model provided comprehensible information regarding the new cases to be predicted, which is an advantage inherent to a decision tree. Initially, the importance of each dependent variable to cost estimation was provided, as shown in Figure 6. These values indicate the importance 
Tree graph for construction cost

Number of nonterminal nodes: 2 , number of terminal nodes: 3

Tree number: 1

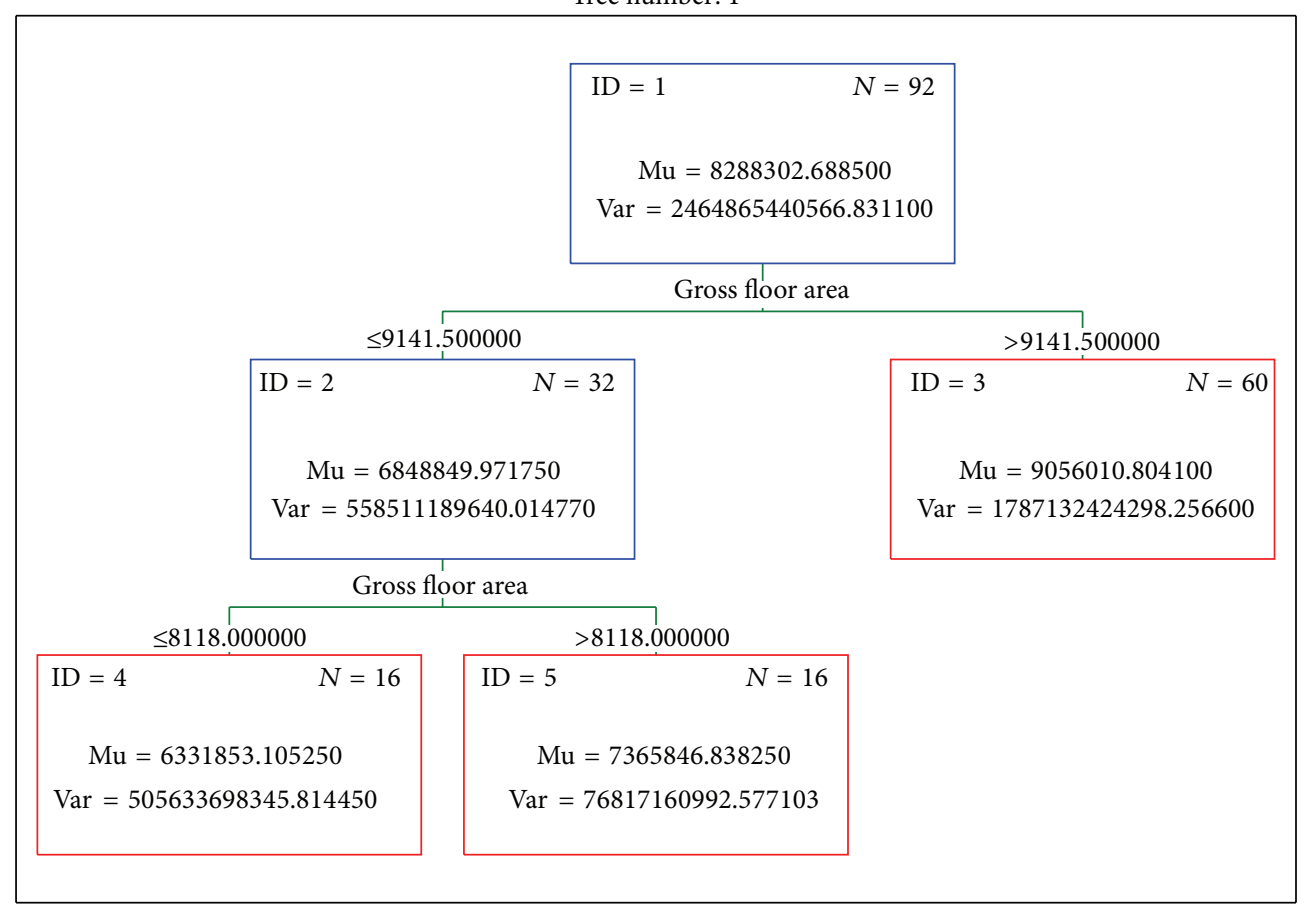

FIGURE 7: An example of structure model.

TABLE 2: Summary of results by estimation model.

\begin{tabular}{lcccc}
\hline \multirow{2}{*}{ Error rate (\%) } & \multicolumn{2}{c}{ NN } & \multicolumn{2}{c}{ BRT } \\
& Fre. (\%) & Cum. (\%) & Fre. (\%) & Cum. (\%) \\
\hline $0.0-2.5$ & $3(10.0)$ & $3(10.0)$ & $6(20.0)$ & $6(20.0)$ \\
$2.5-5.0$ & $11(36.7)$ & $14(46.7)$ & $10(33.3)$ & $16(53.3)$ \\
$5.0-7.5$ & $6(20.0)$ & $20(66.7)$ & $6(20.0)$ & $22(73.3)$ \\
$7.5-10.0$ & $8(26.7)$ & $28(93.3)$ & $2(6.7)$ & $24(80.0)$ \\
$10.0-12.5$ & $1(3.3)$ & $29(96.7)$ & $3(10.0)$ & $27(90.0)$ \\
$12.5-15.0$ & $1(3.3)$ & $30(100)$ & $2(6.7)$ & $29(96.7)$ \\
$15.0-17.5$ & $0(0)$ & $30(100)$ & $1(3.3)$ & $30(100)$ \\
\hline MAERs & 6.05 & - & 5.80 & - \\
\hline
\end{tabular}

of each variable for the construction cost estimation in the model. Finally, the tree structures in the model were provided as shown in Figure 7. This shows the estimation rules, such as the applied variables and their influence on the proposed model. Thus, an intuitive understanding of the whole structure of the model is possible.

5.2. Discussion of Results. This study was conducted using 234 school building construction projects. In addition, 30 of these projects were used for testing. In terms of the estimation accuracy, the BRT model showed slightly better results than the NN model, with MAERs of 5.80 and 6.05, respectively. In terms of the construction cost estimation, it is difficult to conclude that the performance of the BRT model is superior to that of the NN model because the gap between the two is not statistically different. However, even the similar performance of the BRT model is notable because the NN model has proven its superior performance in terms of cost estimation accuracy in previous studies. Similarly, in predicting the software project effort, Elish [43] compared the estimation accuracy of neural network, linear regression, support vector regression (SVR), and BRT. Consequently, BRT outperformed the other techniques in terms of the estimation performance that has been also achieved by SVR. These results mean that the BRT has remarkable performance in regression problem as well as classification one. Moreover, the BRT model provided additional information, that is, an importance plot and structure model, which helps the estimator comprehend the decision making process intuitively.

Consequently, these results reveal that a BRT, which is a new AI approach in the field of construction, has potential applicability in preliminary cost estimations. It can assist estimators in avoiding serious errors in predicting the construction costs when only limited information is available during the early stages of a building construction project. Moreover, a BRT has a large utilization possibility because the boosting approach can employ existing AI techniques such as a NN and SVM, along with decision trees, as base learners during the boosting procedure. 
TABLE 3: Cost estimation results of each test set.

\begin{tabular}{|c|c|c|c|c|c|}
\hline \multirow{2}{*}{ Number } & \multirow{2}{*}{$\begin{array}{c}\text { Historical cost } \\
(1,000 \mathrm{KRW})\end{array}$} & \multicolumn{2}{|c|}{ Neural networks } & \multicolumn{2}{|c|}{ Boosting regression tree } \\
\hline & & $\begin{array}{l}\text { Predicted cost } \\
(1,000 \text { KRW })\end{array}$ & Error rate $(\%)$ & $\begin{array}{l}\text { Predicted cost } \\
(1,000 \mathrm{KRW})\end{array}$ & Error rate $(\%)$ \\
\hline 1 & $6,809,450$ & $7,704,034$ & 13.14 & $7,206,795$ & 5.84 \\
\hline 2 & $9,351,716$ & $10,015,906$ & 7.10 & $9,805,656$ & 4.85 \\
\hline 3 & $6,656,230$ & $7,251,317$ & 8.94 & $6,322,112$ & 5.02 \\
\hline 4 & $7,119,470$ & $7,128,513$ & 0.13 & $7,418,373$ & 4.20 \\
\hline 5 & $7,304,747$ & $7,978,990$ & 9.23 & $7,349,178$ & 0.61 \\
\hline 6 & $9,729,392$ & $9,516,946$ & 2.18 & $9,259,162$ & 4.83 \\
\hline 7 & $10,801,826$ & $9,817,999$ & 9.11 & $9,682,119$ & 10.37 \\
\hline 8 & $7,944,318$ & $7,246,763$ & 8.78 & $7,136,773$ & 10.17 \\
\hline 9 & $10,879,004$ & $10,136,431$ & 6.83 & $10,572,777$ & 2.81 \\
\hline 10 & $7,552,814$ & $7,764,300$ & 2.80 & $7,683,295$ & 1.73 \\
\hline 11 & $8,845,099$ & $8,558,536$ & 3.24 & $8,370,497$ & 5.37 \\
\hline 12 & $10,690,800$ & $10,001,503$ & 6.45 & $10,015,284$ & 6.32 \\
\hline 13 & $8,694,721$ & $8,258,452$ & 5.02 & $8,446,796$ & 2.85 \\
\hline 14 & $6,582,636$ & $6,810,406$ & 3.46 & $6,954,507$ & 5.65 \\
\hline 15 & $7,583,680$ & $8,312,216$ & 9.61 & $8,194,292$ & 8.05 \\
\hline 16 & $7,099,220$ & $7,955,966$ & 12.07 & $8,292,381$ & 16.81 \\
\hline 17 & $8,145,147$ & $8,604,444$ & 5.64 & $8,522,009$ & 4.63 \\
\hline 18 & $8,652,810$ & $7,853,765$ & 9.23 & $8,270,169$ & 4.42 \\
\hline 19 & $10,527,278$ & $10,040,039$ & 4.63 & $9,611,194$ & 8.70 \\
\hline 20 & $6,679,924$ & $6,467,344$ & 3.18 & $7,397,923$ & 10.75 \\
\hline 21 & $8,383,830$ & $9,203,887$ & 9.78 & $8,487,286$ & 1.23 \\
\hline 22 & $7,298,932$ & $8,018,225$ & 9.85 & $8,294,895$ & 13.65 \\
\hline 23 & $7,505,428$ & $7,749,053$ & 3.25 & $7,967,265$ & 6.15 \\
\hline 24 & $7,710,921$ & $7,622,053$ & 1.15 & $7,795,563$ & 1.10 \\
\hline 25 & $6,196,652$ & $6,503,022$ & 4.94 & $5,940,634$ & 4.13 \\
\hline 26 & $8,897,861$ & $8,554,455$ & 3.86 & $8,714,123$ & 2.06 \\
\hline 27 & $7,840,787$ & $8,535,617$ & 8.86 & $8,863,975$ & 13.05 \\
\hline 28 & $8,023,067$ & $7,666,898$ & 4.44 & $6,900,068$ & 14.00 \\
\hline 29 & $7,495,213$ & $7,270,806$ & 2.99 & $7,695,613$ & 2.67 \\
\hline 30 & $7,653,005$ & $8,003,292$ & 4.58 & $7,775,139$ & 1.60 \\
\hline \multicolumn{2}{|c|}{ MAERs } & & 6.05 & & 5.80 \\
\hline
\end{tabular}

TABLE 4: Descriptive analysis of error rate estimation.

\begin{tabular}{lccccc}
\hline \multicolumn{3}{c}{ MAERs Std, deviation Std, error } & \multicolumn{2}{c}{$\begin{array}{c}95 \% \text { confidence interval } \\
\text { of the MAERs } \\
\text { Lower }\end{array}$} & Upper \\
\hline NN & 6.045 & 3.192 & 0.583 & 2.542 & 4.291 \\
BRT & 5.800 & 3.980 & 0.727 & 3.170 & 5.351 \\
\hline
\end{tabular}

\section{Conclusion}

This study applied a BRT to construction cost estimation, that is, the regression problem, to examine the applicability of the boosting approach to a regression problem in the construction domain. To evaluate the performance of the BRT model, its performance was compared with that of an NN model, which had previously proven its high performance capability in the cost estimation domains. The BRT model showed similar results when using 234 actual cost datasets of a building construction project in Korea. Moreover, the BRT model can provide additional information regarding the variables to support estimators in comprehending the decision making process. These results demonstrated that the BRT has dual advantages of boosting and decision trees. The boosting approach has great potential to be a leading technique in next generation construction cost estimation systems.

In this study, an examination using a relatively small dataset and number of variables was carried out on the performance of a BRT for construction cost estimation. Although 
both models performed satisfactorily, further detailed experiments and analyses regarding the quality of the collected data are necessary to utilize the proposed model for an actual project.

\section{Conflict of Interests}

The author declares that there is no conflict of interests regarding the publication of this paper.

\section{Acknowledgment}

This work was supported by Kyonggi University Research Grant 2012.

\section{References}

[1] G.-H. Kim, J.-E. Yoon, S.-H. An, H.-H. Cho, and K.-I. Kang, "Neural network model incorporating a genetic algorithm in estimating construction costs," Building and Environment, vol. 39, no. 11, pp. 1333-1340, 2004.

[2] G. H. Kim, S. H. An, and K. I. Kang, "Comparison of construction cost estimating models based on regression analysis, neural networks, and case-based reasoning," Building and Environment, vol. 39, no. 10, pp. 1235-1242, 2004.

[3] G. H. Kim and S. H. An, "A study on the correlation between selection methods of input variables and number of data in estimating accuracy: cost estimating using neural networks in apartment housing projects," Journal of the Architectural Institute of Korea, vol. 23, no. 4, pp. 129-137, 2007.

[4] H.-G. Cho, K.-G. Kim, J.-Y. Kim, and G.-H. Kim, "A comparison of construction cost estimation using multiple regression analysis and neural network in elementary school project," Journal of the Korea Institute of Building Construction, vol. 13, no. 1, pp. 66-74, 2013.

[5] G. H. Kim, J. M. Shin, S. Kim, and Y. Shin, "Comparison of school building construction costs estimation methods using regression analysis, neural network, and support vector machine," Journal of Building Construction and Planning Research, vol. 1, no. 1, pp. 1-7, 2013.

[6] S. H. An and K. I. Kang, "A study on predicting construction cost of apartment housing using experts' knowledge at the early stage of projects," Journal of the Architectural Institute of Korea, vol. 21, no. 6, pp. 81-88, 2005.

[7] H. Brink, Real-World Machine Learning, Manning, 2014.

[8] R. A. McKim, "Neural network applications to cost engineering," Cost Engineering, vol. 35, no. 7, pp. 31-35, 1993.

[9] I.-C. Yeh, "Quantity estimating of building with logarithmneuron networks," Journal of Construction Engineering and Management, vol. 124, no. 5, pp. 374-380, 1998.

[10] J. Bode, "Neural networks for cost estimation: simulations and pilot application," International Journal of Production Research, vol. 38, no. 6, pp. 1231-1254, 2000.

[11] S. K. Kim and I. W. Koo, "A neural network cost model for office buildings," Journal of the Architectural Institute of Korea, vol. 16, no. 9, pp. 59-67, 2000.

[12] M.-Y. Cheng and Y.-W. Wu, "Construction conceptual cost estimates using support vector machine," in Proceedings of the 22nd International Symposium on Automation and Robotics in Construction (ISARC '05), Ferrara, Italy, September 2005.
[13] U. Y. Park and G. H. Kim, "A study on predicting construction cost of apartment housing projects based on support Vector regression at the early project stage," Journal of the Architectural Institute of Korea, vol. 23, no. 4, pp. 165-172, 2007.

[14] S. H. An, K. I. Kang, M. Y. Cho, and H. H. Cho, "Application of support vector machines in assessing conceptual cost estimates," Journal of Computing in Civil Engineering, vol. 21, no. 4, pp. 259-264, 2007.

[15] F. Kong, X. Wu, and L. Cai, "Application of RS-SVM in construction project cost forecasting," in Proceedings of the 4th International Conference on Wireless Communications, Networking and Mobile Computing (WiCOM '08), Dalian, China, October 2008.

[16] J.-M. Shin and G.-H. Kim, "A study on predicting construction cost of educational building project at early stage using support vector machine technique," The Journal of Educational Environment Research, vol. 11, no. 3, pp. 46-54, 2012.

[17] J. M. de la Garza and K. G. Rouhana, "Neural networks versus parameter-based applications in cost estimating," Cost Engineering, vol. 37, no. 2, pp. 14-18, 1995.

[18] R. Creese and L. Li, "Cost estimation of timber bridge using neural networks," Cost Engineering, vol. 37, no. 5, pp. 17-22, 1995.

[19] H. Adeli and M. Wu, "Regularization neural network for construction cost estimation," Journal of Construction Engineering and Management, vol. 124, no. 1, pp. 18-24, 1998.

[20] T. M. S. Elhag and A. H. Boussabaine, "An artificial neural system for cost estimation of construction projects," in Proceedings of the 14th ARCOM Annual Conference, September 1998.

[21] M. W. Emsley, D. J. Lowe, A. R. Duff, A. Harding, and A. Hickson, "Data modelling and the application of a neural network approach to the prediction of total construction costs," Construction Management and Economics, vol. 20, no. 6, pp. 465-472, 2002.

[22] V. N. Vapnik, The Nature of Statistical Learning Theory, Springer, London, UK, 1999.

[23] M. Hongwei, "An improved support vector machine based on rough set for construction cost prediction," in Proceedings of the International Forum on Computer Science-Technology and Applications (IFCSTA '09), December 2009.

[24] M. Y. Cheng, H. S. Peng, Y. W. Wu, and T. L. Chen, "Estimate at completion for construction projects using evolutionary support vector machine inference model," Automation in Construction, vol. 19, no. 5, pp. 619-629, 2010.

[25] P. R. Kumar and V. Ravi, "Bankruptcy prediction in banks and firms via statistical and intelligent techniques: a review," European Journal of Operational Research, vol. 180, no. 1, pp. 128, 2007.

[26] Y. Freund and R. E. Schapire, "A decision-theoretic generalization of on-line learning and an application to boosting," Journal of Computer and System Sciences, vol. 55, no. 1, part 2, pp. 119139, 1997.

[27] D. Arditi and T. Pulket, "Predicting the outcome of construction litigation using boosted decision trees," Journal of Computing in Civil Engineering, vol. 19, no. 4, pp. 387-393, 2005.

[28] E. Osuna, R. Freund, and F. Girosi, "Training support vector machines: an application to face detection," in Proceedings of the IEEE Computer Society Conference on Computer Vision and Pattern Recognition, pp. 130-136, San Juan, Puerto Rico, USA, June 1997.

[29] C. P. Papageorgiou, M. Oren, and T. Poggio, "A general framework for object detection," in Proceedings of the IEEE 
6th International Conference on Computer Vision, pp. 555-562, January 1998.

[30] R. E. Schapire, Y. Freund, P. Bartlett, and W. S. Lee, "Boosting the margin: a new explanation for the effectiveness of voting methods," The Annals of Statistics, vol. 26, no. 5, pp. 1651-1686, 1998.

[31] Y. Shin, D. W. Kim, J. Y. Kim, K. I. Kang, M. Y. Cho, and H. H. Cho, "Application of adaboost to the retaining wall method selection in construction," Journal of Computing in Civil Engineering, vol. 23, no. 3, pp. 188-192, 2009.

[32] E. Alfaro, N. García, M. Gámez, and D. Elizondo, "Bankruptcy forecasting: an empirical comparison of AdaBoost and neural networks," Decision Support Systems, vol. 45, no. 1, pp. 110-122, 2008.

[33] E. A. Park, A comparison of SVM and boosting methods and their application for credit scoring [M.S. thesis], Seoul National University, 2005.

[34] Y. Freund and R. E. Schapire, "A short introduction to boosting," Journal of Japanese Society for Artificial Intelligence, vol. 14, no. 5, pp. 771-780, 1999.

[35] Y.-S. Lee, H.-J. Oh, and M.-K. Kim, "An empirical comparison of bagging, boosting and support vector machine classifiers in data mining," Korean Journal of Applied Statistics, vol. 18, no. 2, pp. 343-354, 2005.

[36] Y. Shin, T. Kim, H. Cho, and K. I. Kang, "A formwork method selection model based on boosted decision trees in tall building construction," Automation in Construction, vol. 23, pp. 47-54, 2012.

[37] J. H. Friedman, "Stochastic gradient boosting," Computational Statistics \& Data Analysis, vol. 38, no. 4, pp. 367-378, 2002.

[38] J. Raftery, "The state of cost/modelling in the UK construction industry: a multi criteria approach," in Building Cost Modeling and Computers, P. S. Brandon, Ed., pp. 49-71, E\&PN Spon, London, UK, 1987.

[39] G. H. Kim, Construction cost prediction system based on artificial intelligence at the project planning stage [Ph.D. thesis], Korea University, Seoul, Republic of Korea, 2004.

[40] G. Ridgeway, "Generallized boosted model: A guide to the gbm package," CiteSeerx, 2005, http://citeseerx.ist.psu.edu/viewdoc/ summary?doi=10.1.1.151.4024.

[41] A. M. Filippi, İ. Güneralp, and J. Randall, "Hyperspectral remote sensing of aboveground biomass on a river meander bend using multivariate adaptive regression splines and stochastic gradient boosting," Remote Sensing Letters, vol. 5, no. 5, pp. 432-441, 2014.

[42] D. C. Carslaw and P. J. Taylor, "Analysis of air pollution data at a mixed source location using boosted regression trees," Atmospheric Environment, vol. 43, no. 22-23, pp. 3563-3570, 2009.

[43] M. O. Elish, "Improved estimation of software project effort using multiple additive regression trees," Expert Systems with Applications, vol. 36, no. 7, pp. 10774-10778, 2009.

[44] M. P. Martin, D. L. Seen, L. Boulonne et al., "Optimizing pedotransfer functions for estimating soil bulk density using boosted regression trees," Soil Science Society of America Journal, vol. 73, no. 2, pp. 485-493, 2009.

[45] R. Ismail and O. Mutanga, "A comparison of regression tree ensembles: predicting Sirex noctilio induced water stress in Pinus patula forests of KwaZulu-Natal, South Africa," International Journal of Applied Earth Observation and Geoinformation, vol. 12, no. 1, pp. S45-S51, 2010.
[46] T. J. Hastie, R. Tibshirani, and J. Friedman, The Elements of Statistical Learning, Springer, New York, NY, USA, 2nd edition, 2009.

[47] R. E. Schapire, "A brief introduction to boositng," in Proceedings of the 16th International Joint Conference on Artificial Intelligence (IJCAI '99), vol. 2, pp. 1401-1406, Stockholm, Sweden, JulyAugust 1999.

[48] J. Friedman, T. Hastie, and R. Tibshirani, "Additive statistical regression: a statistical view of boosting," The Annals of Statistics, vol. 28, pp. 337-407, 2000.

[49] H. Drucker, "Improving regressors using boosting techniques," in Proceedings of the 14th International Conference on Machine Learning, Nashville, Tenn, USA, July 1997.

[50] L. Breiman, "Prediction games and arcing algorithms," Neural Computation, vol. 11, no. 7, pp. 1493-1517, 1999.

[51] G. Ridgeway, D. Madigan, and T. Richardson, "Boosting methodology for regression problems," in Proceedings of the 7th International Workshop on Artificial Intelligence and Statistics, Fort Lauderdale, Fla, USA, January 1999.

[52] J. H. Friedman, "Greedy function approximation: a gradient boosting machine," The Annals of Statistics, vol. 29, no. 5, pp. 1189-1232, 2001.

[53] J. Ye, J.-H. Chow, J. Chen, and Z. Zheng, "Stochastic gradient boosted distributed decision trees," in Proceedings of the ACM 18th International Conference on Information and Knowledge Management (CIKM '09), pp. 2061-2064, Hong Kong, November 2009 .

[54] J. H. Friedman and J. J. Meulman, "Multiple additive regression trees with application in epidemiology," Statistics in Medicine, vol. 22, no. 9, pp. 1365-1381, 2003.

[55] M. Skitmore, "The effect of project information on the accuracy of building price forecasts," in Building Cost Modeling and Computers, P. S. Brandon, Ed., E \& FN SPON, London, UK, 1987.

[56] M. Skitmore, "Early stage construction price forecasting: a review of performance," Occasional Paper, Royal Institute of Chartered Surveyors, London, UK, 1991.

[57] T. Hill and P. Lewicki, STATISTICS: Methods and Applications, StatSoft, Tulsa, Okla, USA, 1st edition, 2006. 

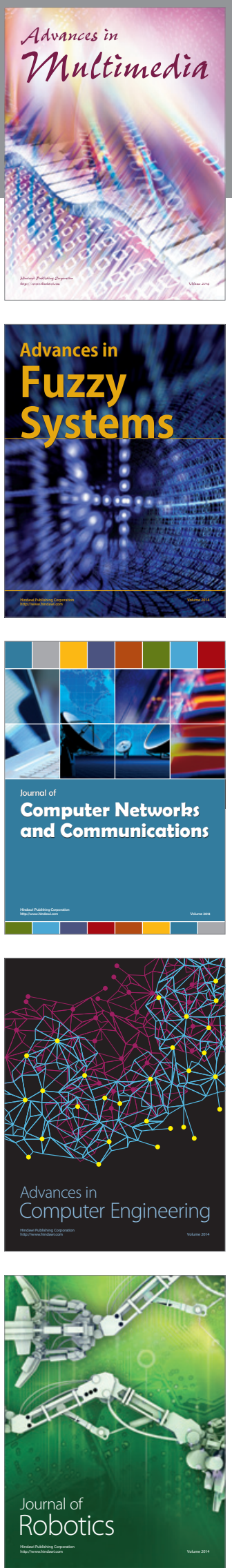

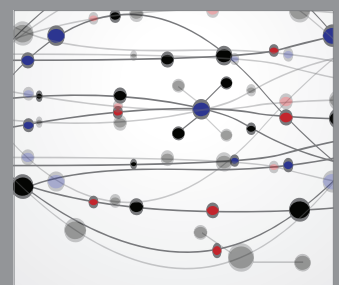

The Scientific World Journal
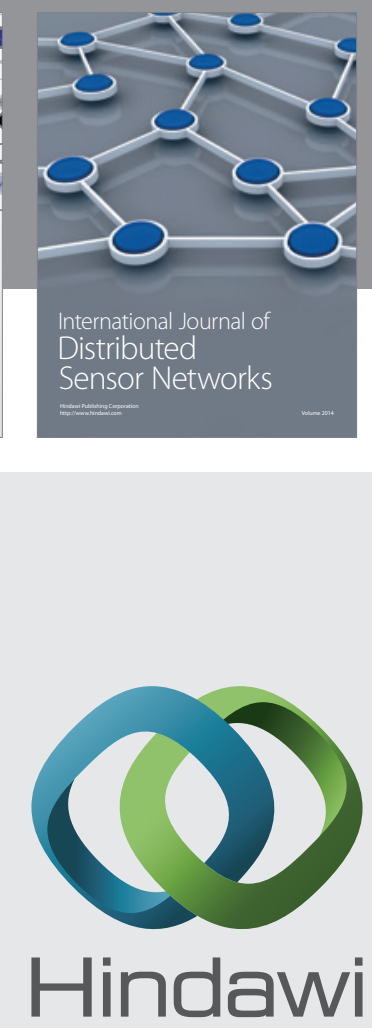

Submit your manuscripts at

http://www.hindawi.com
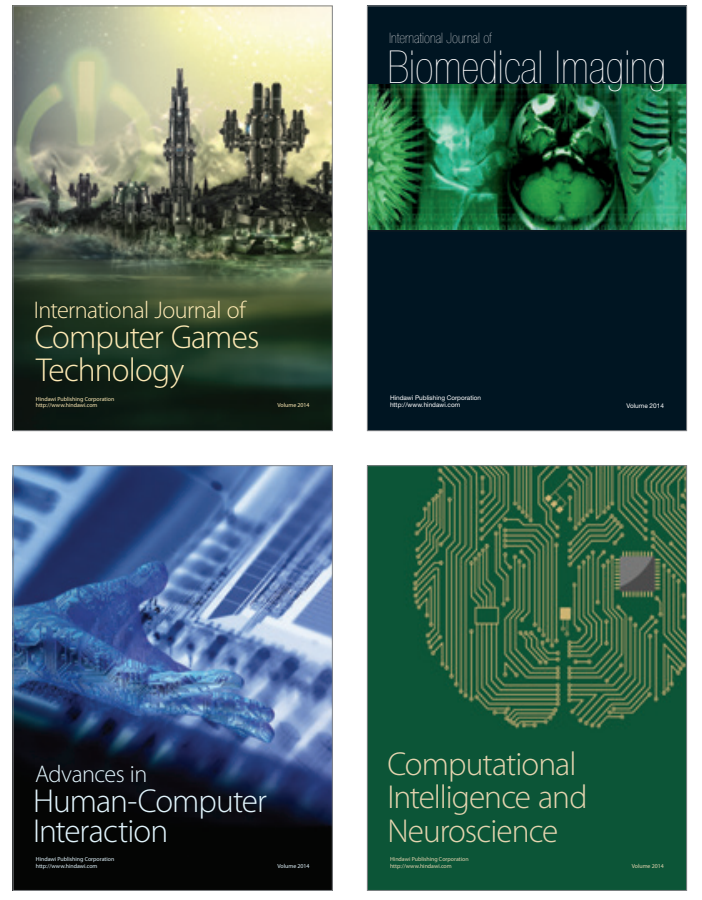
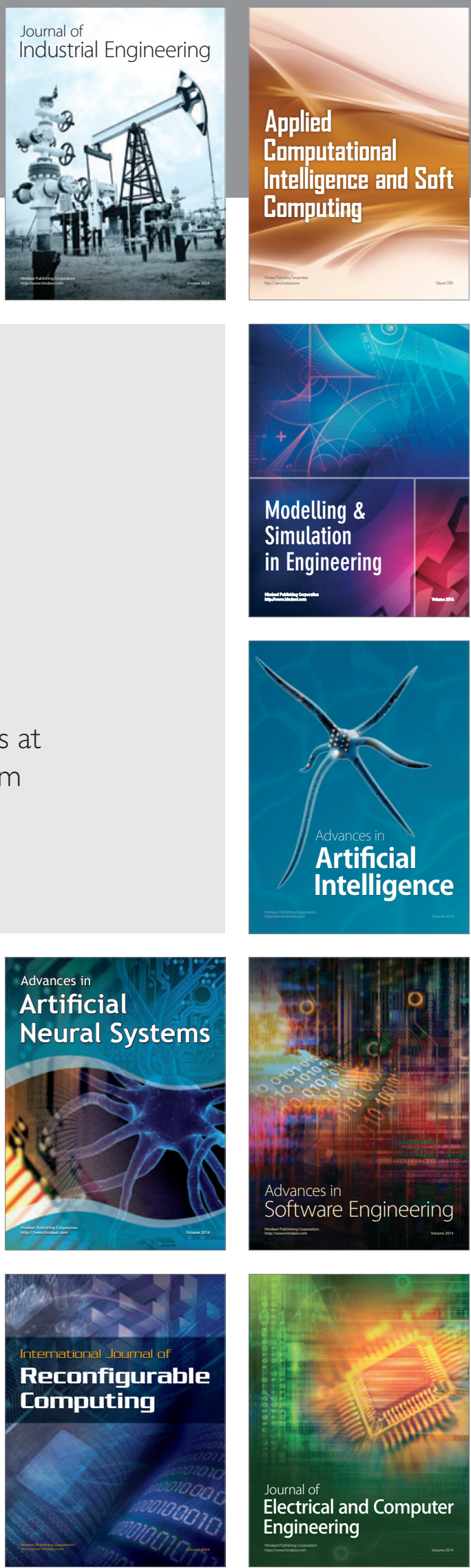